\title{
On conformal submersions with geodesic or minimal fibers
}

\author{
Tomasz Zawadzki ${ }^{1}$ iD
}

Received: 9 January 2020 / Accepted: 3 June 2020 / Published online: 24 June 2020

(c) The Author(s) 2020

\begin{abstract}
We prove that every conformal submersion from a round sphere onto an Einstein manifold with fibers being geodesics is-up to an isometry-the Hopf fibration composed with a conformal diffeomorphism of the complex projective space of appropriate dimension. We also show that there are no conformal submersions with minimal fibers between manifolds satisfying certain curvature assumptions.
\end{abstract}

Keywords Conformal submersions $\cdot$ Foliations $\cdot$ Einstein metrics

Mathematics Subject Classification $53 \mathrm{C} 12 \cdot 53 \mathrm{C} 21 \cdot 53 \mathrm{C} 20$

\section{Introduction}

One of the common problems of Riemannian geometry and theory of foliations is the existence of foliations and distributions, satisfying certain geometric properties, on a given Riemannian manifold. Examining the natural representation of the product of orthogonal groups yields several interesting classes of distributions [14], described by their extrinsic geometry: totally geodesic, totally umbilical, and minimal. When the orthogonal distribution to the foliation belongs to one of these classes, we call the foliation Riemannian, conformal, or an $\operatorname{SL}(q)$-foliation (respectively), where $q$ is the dimension of the distribution. Of special interest are those foliations for which both distributions, tangent and orthogonal to the leaves, belong to one (not necessarily the same) of the families in the above classification. The aim of this paper is to study one such case-of a conformal foliation with minimal fibers, with a particular focus on conformal fibrations of spheres (here always considered "round", i.e., equipped with the standard Riemannian metric induced from Euclidean space) by great circles.

Fibrations of spheres by great circles have been thoroughly examined from the topological and differential point of view [6,7]. It was established that they exist only on spheres of odd dimension, the leaf space of such fibration is diffeomorphic to the complex projective space of appropriate dimension [12], and that any such fibration deformation retracts

Tomasz Zawadzki

tomasz.zawadzki@wmii.uni.lodz.pl

1 Faculty of Mathematics and Computer Science, University of Lodz, Banacha 22, 90-238 Lodz, Poland 
to the Hopf fibration [15]. Fibrations of spheres, satisfying additional geometric assumption: giving rise to a Riemannian foliation by their fibers, were examined in [4, 18, 22]. A generalization of this assumption-when the fibration defines a conformal foliation, and its one-dimensional fibers are not necessarily great circles-was examined on the threedimensional sphere in [10]. Due to these works, Riemannian submersions from spheres and conformal submersions from the 3-sphere are fully classified from the point of view of Riemannian geometry. They all can be related to the Hopf fibration by a pair of isometries (in Riemannian foliation case) or conformal diffeomorphisms (in the case of a conformal foliation of $S^{3}$ ) of their domain and image.

In this paper, we examine conformal submersions from round spheres of any odd dimension (greater than 3), with fibers being great circles, using direct methods of Riemannian geometry, introduced in [16], and applied to conformal submersions in [9]. This approach seems to require some assumptions on the geometry of the image of the submersion. Due to existence of solutions of the Yamabe problem [1, 19, 20,23], one can freely assume that the image has constant scalar curvature - up to a conformal change of the metric. In this paper, a stronger assumption is considered - that the image of the submersion is conformal to an Einstein manifold. Thus, instead of conformally constant scalar curvature, we assume what might be viewed as a one-step stronger condition [2] - that some metric in the conformal class of the image has constant Ricci curvature.

We prove that the only Einstein manifold, that can be the image of a conformal submersion from the sphere with fibers being great circles, is isometric to the complex projective space with the Fubini-Study metric. Moreover, in this case, the submersion is the composition of the Hopf fibration and a conformal diffeomorphism of the complex projective space. In the second part of the paper, we examine conformal submersions with minimal fibers and find some restrictions on the scalar curvature of their image. These restrictions apply in particular to manifolds with metrics of constant scalar curvature, and, hence, can be expressed in terms of conformal classes of Riemannian metrics.

\section{Definitions}

Let $(M, g),\left(B, g_{\mathrm{B}}\right)$ be smooth Riemannian manifolds, of dimensions $m, b$ (resp.) satisfying inequality $m \geq b$. Let $\pi:(M, g) \rightarrow\left(B, g_{\mathrm{B}}\right)$ be a submersion, i.e., a smooth mapping of maximal rank. For every $q \in B$, the set $\pi^{-1}(q)$ is a submanifold of $M$, called the fiber of $\pi$ over $q$. All the fibers of submersion $\pi$ are submanifolds of dimension $k=m-b$. Elements of $T M$ tangent to the fibers form the smooth, integrable vertical distribution; its orthogonal complement with respect to $g$ is called the horizontal distribution. Vectors and vector fields with values in the horizontal (resp. vertical) distribution will be called horizontal (resp. vertical). Orthogonal (with respect to $g$ ) projections onto the horizontal and vertical distributions will be denoted by $\mathcal{H}$ and $\mathcal{V}$, respectively.

Definition 1 Let $(M, g),\left(B, g_{\mathrm{B}}\right)$ be smooth Riemannian manifolds. A submersion $\pi:(M, g) \rightarrow\left(B, g_{\mathrm{B}}\right)$ is called conformal (or: horizontally conformal) if its differential $\pi_{*}$ restricted to the horizontal distribution of $\pi$ is a conformal map, i.e., there exists a function $f \in C^{\infty}(M)$, such that for all horizontal vectors $X, Y$, we have:

$$
e^{-2 f} g(X, Y)=g_{\mathrm{B}}\left(\pi_{*} X, \pi_{*} Y\right) .
$$


The function $f$ in the above equation will be called the dilation of submersion $\pi$; a conformal submersion with $f=0$ is called a Riemannian submersion [16].

In what follows, $\nabla$ will always denote the Levi-Civita connection on $M$ and $R$-its curvature tensor, with convention $R(X, Y) Z=\nabla_{X} \nabla_{Y} Z-\nabla_{Y} \nabla_{X} Z-\nabla_{[X, Y]} Z$. For all $\phi \in C^{\infty}(M)$, we denote by $\nabla \phi$ the gradient (with respect to $g$ ) of the function $\phi$, i.e., $g(\nabla \phi, X)=X(\phi)$ for all $X \in T M$, and by Hess $\phi$ its hessian, $\operatorname{Hess} \phi(X, Y)=g\left(\nabla_{X} \nabla \phi, Y\right)=g\left(\nabla_{Y} \nabla \phi, X\right)$ for all $X, Y \in T M$. Following O'Neill [16], we define a $(1,2)$-tensor $A$ by the formula:

$$
A_{E} F=\mathcal{V} \nabla_{\mathcal{H} E} \mathcal{H} F+\mathcal{H} \nabla_{\mathcal{H} E} \mathcal{V} F
$$

where $E, F$ are any smooth vector fields on $M$ (one can show that the value of $A$ at a point depends only on the values of these fields at that point). Recall that for all vectors $E$, the $(1,1)$ tensor $A_{E}$ is antisymmetric with respect to $g$, i.e., $g\left(A_{E} F, G\right)=-g\left(A_{E} G, F\right)$ for all $E, F, G \in T M$. For all horizontal vectors $X, Y$, we have the following formula [9]:

$$
A_{X} Y=\frac{1}{2} \mathcal{V}[X, Y]-g(X, Y) \mathcal{V} \nabla f .
$$

Definition 2 Let $\pi:(M, g) \rightarrow\left(B, g_{\mathrm{B}}\right)$ be a submersion, let $\left\{X_{1}, \ldots, X_{\mathrm{b}}\right\}$ be an orthonormal basis of the horizontal distribution on $M$ at the point $p,\left\{U_{1}, \ldots, U_{k}\right\}$ be an orthonormal basis of the vertical distribution at $p$, and $\left\{Y_{1}, \ldots, Y_{\mathrm{b}}\right\}$ be an orthonormal basis of the tangent space of $B$ at the point $q$. Let $R_{\mathrm{B}}$ denote the curvature tensor of the Levi-Civita connection on $\left(B, g_{\mathrm{B}}\right)$. We define the quantities:

$$
\begin{aligned}
S_{\text {mix }}(p) & =\sum_{i=1}^{k} \sum_{j=1}^{b} g\left(R\left(U_{i}, X_{j}\right) X_{j}, U_{i}\right) \\
K_{\mathcal{H}}(p) & =\sum_{i, j=1}^{b} g\left(R\left(X_{i}, X_{j}\right) X_{j}, X_{i}\right) \\
K_{\mathrm{B}}(q) & =\sum_{i, j=1}^{b} g_{\mathrm{B}}\left(R_{\mathrm{B}}\left(Y_{i}, Y_{j}\right) Y_{j}, Y_{i}\right) .
\end{aligned}
$$

We will call $S_{\text {mix }}$ the mixed scalar curvature of $M$ and $K_{\mathcal{H}}$ the horizontal scalar curvature of $M ; K_{\mathrm{B}}$ is the scalar curvature of $B$.

For linearly independent vectors $X, Y \in T_{p} M$, the sectional curvature of the tangent plane $X \wedge Y$ is defined as:

$$
\sec (X, Y)=\frac{g(R(X, Y) Y, X)}{g(X, X) g(Y, Y)-g(X, Y)^{2}} .
$$

For vectors $X_{\mathrm{B}}, Y_{\mathrm{B}} \in T_{q} B$, we analogously compute the sectional curvature of the tangent plane $X_{\mathrm{B}} \wedge Y_{\mathrm{B}}$ and denote it by $\sec _{\mathrm{B}}\left(X_{\mathrm{B}}, Y_{\mathrm{B}}\right)$.

For all $\phi \in C^{\infty}(M)$, analogously to the Laplacian:

$$
\Delta \phi=\sum_{i=1}^{n} \operatorname{Hess} \phi\left(E_{i}, E_{i}\right),
$$


where $E_{i}$ form an orthonormal basis of the tangent space at a point, we introduce the Laplacian along fibers of $\pi$, defined by the formula:

$$
\Delta^{\mathcal{V}} \phi=\sum_{i=1}^{k} g\left(\nabla_{U_{i}} \mathcal{V} \nabla \phi, U_{i}\right),
$$

where $\left\{U_{1}, \ldots, U_{k}\right\}$ form an orthonormal basis of the vertical distribution at a point.

The Ricci tensor of a manifold $\left(B, g_{\mathrm{B}}\right)$ will be denoted by $\operatorname{Ric}_{\mathrm{B}}$. Recall that $\left(B, g_{\mathrm{B}}\right)$ is Einstein if there exists $\lambda_{\mathrm{B}} \in \mathbb{R}$, such that $\mathrm{Ric}_{\mathrm{B}}=\lambda_{\mathrm{B}} g_{\mathrm{B}}$.

In Sect. 4, we will use the following tensor, describing extrinsic geometry of the fibers of a submersion.

Definition 3 Let $\pi:(M, g) \rightarrow\left(B, g_{\mathrm{B}}\right)$ be a submersion. We define the second fundamental form of the fibers as the $(1,2)$-tensor $h$ given by:

$$
h(U, V)=\mathcal{H} \nabla_{U} V
$$

for all vertical $U, V$.

Note that $h(U, V)=h(V, U)$. We define the mean curvature vector field $H$ of the fibers, the value of which at $p \in M$ is given by the formula $H=\sum_{i=1}^{k} h\left(U_{i}, U_{i}\right)$, for an orthonormal basis $\left\{U_{1}, \ldots, U_{k}\right\}$ of the vertical distribution at $p$. We call the fibers of a submersion minimal if $H=0$ on $M$. If $h(U, V)=\frac{1}{k} g(U, V) H$ for all vertical $U, V$, then the fibers are called totally umbilical.

We will also use the following notation for the norm of a vector: $\|E\|=\sqrt{g(E, E)}$ for every vector $E$ on a Riemannian manifold $(M, g)$.

\section{Conformal submersions from spheres with fibers being geodesics}

We consider a conformal submersion from a sphere, the fibers of which are geodesics, and relate this submersion to the Hopf fibration. We consider only odd-dimensional spheres, as by topological arguments only those admit such submersions. Indeed, by the Ehresmann theorem, every submersion from a sphere defines a fibration [3]. A short argument [13] shows that a circle fibration defines a unit vector field on a 2-1 cover of the sphere (using a unit vector field tangent to fibers, determined up to sign), and hence-for spheres of dimension at least 2-a unit vector field on the sphere itself, as it is simply connected. Due to the Euler characteristic, such vector field can exist on a sphere of odd-dimension only.

In this section, $g$ will always denote the round metric on a sphere.

Lemma 1 Let $\pi:\left(S^{2 n+1}, g\right) \rightarrow\left(B, g_{\mathrm{B}}\right), n>1$, be a conformal submersion with fibers being geodesics and let $\left(B, g_{\mathrm{B}}\right)$ be an Einstein manifold. Then, there exists a function $\phi \in C^{\infty}\left(S^{2 n+1}\right)$, such that for all unit, horizontal $X \in T S^{2 n+1}$, we have:

$$
\phi=\operatorname{Hess} f(X, X)+(X(f))^{2} .
$$

Proof All the formulas in this proof will be considered at a point $p \in M$, but can be easily seen independent on the choice of that point. For $X, Y \in T_{p} M$-orthonormal, horizontal vectors, we have: [24] 


$$
\begin{aligned}
\sec (X, Y)= & -\operatorname{Hess} f(X, X)-\operatorname{Hess} f(Y, Y) \\
& +\|\nabla f\|^{2}-(X(f))^{2}-(Y(f))^{2} \\
& +e^{-2 f} \sec _{\mathrm{B}}\left(\pi_{*} X, \pi_{*} Y\right)-3\left\|A_{X} Y\right\|^{2} .
\end{aligned}
$$

The above equation can be obtained from the formula for a conformal change of the sectional curvature and the curvature relations for a Riemannian submersion [16].

Let $X_{1}, \ldots, X_{2 n}$ be an orthonormal basis of the horizontal distribution at $p \in S^{2 n+1}$. Then, for all $i \in\{1, \ldots, 2 n\}$, we have:

$$
\begin{aligned}
\sum_{j \neq i} \sec \left(X_{i}, X_{j}\right)= & -(2 n-1) \operatorname{Hess} f\left(X_{i}, X_{i}\right)-\sum_{j \neq i} \operatorname{Hess} f\left(X_{j}, X_{j}\right) \\
& +(2 n-1)\|\nabla f\|^{2}-(2 n-1)\left(X_{i}(f)\right)^{2}-\sum_{j \neq i}\left(X_{j}(f)\right)^{2} \\
& +e^{-2 f} \sum_{j \neq i} \sec _{\mathrm{B}}\left(\pi_{*} X_{i}, \pi_{*} X_{j}\right)-3 \sum_{j \neq i}\left\|A_{X_{i}} X_{j}\right\|^{2},
\end{aligned}
$$

where the sums are taken over all vectors of the orthonormal basis except $X_{i}$.

Let $U \in T_{p} M$ be a unit vertical vector. Observe that since $A_{X_{i}} X_{j}$ is vertical and the vertical distribution is spanned by $U$, we have:

$$
\sum_{j \neq i}\left\|A_{X_{i}} X_{j}\right\|^{2}=\sum_{j \neq i} g\left(A_{X_{i}} X_{j}, U\right) g\left(A_{X_{i}} X_{j}, U\right)=\sum_{j \neq i} g\left(A_{X_{i}} X_{j}, U\right)^{2}
$$

also, since $A_{X_{i}} U$ is horizontal and $A_{X_{i}}$ is antisymmetric, from (3), we obtain:

$$
\begin{aligned}
\left\|A_{X_{i}} U\right\|^{2}= & \sum_{j \neq i} g\left(A_{X_{i}} U, X_{j}\right)^{2}+g\left(A_{X_{i}} U, X_{i}\right)^{2} \\
& =\sum_{j \neq i} g\left(A_{X_{i}} X_{j}, U\right)^{2}+g\left(A_{X_{i}} X_{i}, U\right)^{2} \\
& =\sum_{j \neq i} g\left(A_{X_{i}} X_{j}, U\right)^{2}+(U(f))^{2} .
\end{aligned}
$$

Since $\pi$ is a conformal submersion, $\pi_{*} X_{i}$ and $\pi_{*} X_{j}$ are orthogonal on $\left(B, g_{\mathrm{B}}\right)$ for $i \neq j$. As $\left(B, g_{\mathrm{B}}\right)$ is Einstein, we have:

$$
\sum_{j \neq i} \sec _{\mathrm{B}}\left(\pi_{*} X_{i}, \pi_{*} X_{j}\right)=\operatorname{Ric}_{\mathrm{B}}\left(\frac{\pi_{*} X_{i}}{\left\|\pi_{*} X_{i}\right\|}, \frac{\pi_{*} X_{i}}{\left\|\pi_{*} X_{i}\right\|}\right)=\lambda_{\mathrm{B}} g_{\mathrm{B}}\left(\frac{\pi_{*} X_{i}}{\left\|\pi_{*} X_{i}\right\|}, \frac{\pi_{*} X_{i}}{\left\|\pi_{*} X_{i}\right\|}\right)=\lambda_{B},
$$

while on $S^{2 n+1}$ :

$$
\sum_{j \neq i} \sec \left(X_{i}, X_{j}\right)=2 n-1
$$

Using the above with $\Delta f=\sum_{j=1}^{2 n} \operatorname{Hess} f\left(X_{j}, X_{j}\right)+\operatorname{Hess} f(U, U) \quad$ and $\|\nabla f\|^{2}=\sum_{j=1}^{2 n}\left(X_{j}(f)\right)^{2}+(U(f))^{2}$ in $(6)$, we can obtain the following: 


$$
\begin{aligned}
2 n-1= & -(2 n-2) \operatorname{Hess} f\left(X_{i}, X_{i}\right)-\Delta f \\
& +(2 n-2)\|\nabla f\|^{2}-(2 n-2)\left(X_{i}(f)\right)^{2} \\
& +e^{-2 f} \lambda_{\mathrm{B}}-3\left\|A_{X_{i}} U\right\|^{2} \\
& +\operatorname{Hess} f(U, U)+4(U(f))^{2} .
\end{aligned}
$$

From the curvature formula for totally geodesic fibers and directions: vertical and horizontal [24], we obtain:

$$
\sec \left(X_{i}, U\right)=-g\left(\nabla_{U} \mathcal{V} \nabla f, U\right)+\left\|A_{X_{i}} U\right\|^{2}-2(U(f))^{2} .
$$

However, for fibers being geodesics, we have:

$$
\begin{aligned}
g\left(\nabla_{U} \mathcal{V} \nabla f, U\right) & =g\left(\nabla_{U} \nabla f, U\right)-g\left(\nabla_{U} \mathcal{H} \nabla f, U\right) \\
& =\operatorname{Hess} f(U, U)-g\left(\nabla f, \mathcal{H} \nabla_{U} U\right)=\operatorname{Hess} f(U, U),
\end{aligned}
$$

and on the sphere $\sec \left(X_{i}, U\right)=1$. Using this together with (7) and (8) yields:

$$
\begin{aligned}
(2 n-2)\left(\operatorname{Hess} f\left(X_{i}, X_{i}\right)+\left(X_{i}(f)\right)^{2}\right)= & (2 n-2)\|\nabla f\|^{2}-\Delta f \\
& -2 \operatorname{Hess} f(U, U)-2(U(f))^{2} \\
& +e^{-2 f} \lambda_{B}-(2 n+2) .
\end{aligned}
$$

Note that the above equation does not depend on the choice of the unit horizontal vector $X_{i}$. Hence, there exists a function $\phi \in C^{\infty}\left(S^{2 n+1}\right)$ - the right-hand side of (9) - depending only on the dilation of $f$, such that for all unit, horizontal $X$ equation (4) holds.

Proposition 1 Let $\pi:\left(S^{2 n+1}, g\right) \rightarrow\left(B, g_{\mathrm{B}}\right), n>1$, be a conformal submersion with fibers being geodesics and let $\left(B, g_{\mathrm{B}}\right)$ be Einstein manifold. Then, the dilation of $\pi$ is constant along the fibers of $\pi$.

Proof Let $\pi:\left(S^{2 n+1}, g\right) \rightarrow\left(B, g_{\mathrm{B}}\right), n>1$, be a conformal submersion with fibers being geodesics. Using Lemma 1, we can write (5) for all orthonormal, horizontal vectors $X, Y \in T_{p} M$ as:

$$
\begin{aligned}
\sec (X, Y)= & -2 \phi+\|\nabla f\|^{2}-3\left\|A_{X} Y\right\|^{2} \\
& +e^{-2 f} \sec _{\mathrm{B}}\left(\pi_{*} X, \pi_{*} Y\right) .
\end{aligned}
$$

Let $T_{U}^{\sharp}$ be defined by the formula $g\left(T_{U_{1}}^{\sharp}, Y\right)=g\left(\frac{1}{2} \mathcal{V}[X, Y], U\right)$. Then, for orthonormal, horizontal $X, Y$, we have from (3) $A_{X} Y=\frac{1}{2} \mathcal{V}[X, Y]$, and hence:

$$
\left\|A_{X} Y\right\|^{2}=g\left(A_{X} Y, U\right) g\left(A_{X} Y, U\right)=g\left(\frac{1}{2} \mathcal{V}[X, Y], U\right)^{2}=g\left(T_{U}^{\sharp} X, Y\right)^{2} .
$$

Using the fact that $S^{2 n+1}$ has constant curvature 1, we can write (10) as:

$$
\begin{aligned}
\sec _{\mathrm{B}}\left(\pi_{*} X, \pi_{*} Y\right)= & e^{2 f}+2 e^{2 f} \phi-e^{2 f}\|\nabla f\|^{2} \\
& +3 e^{2 f} g\left(T_{U}^{\sharp} X, Y\right)^{2} .
\end{aligned}
$$

Let $X$ be a unit horizontal vector at $p \in M$. Let us introduce the following notation: 


$$
S(X)=\max _{Y \in \mathcal{H}_{p}} \sec _{\mathrm{B}}\left(\pi_{*} X, \pi_{*} Y\right), \quad s(X)=\min _{Y \in \mathcal{H}_{p}} \sec _{\mathrm{B}}\left(\pi_{*} X, \pi_{*} Y\right) .
$$

If $T_{U}^{\sharp} X \neq 0$, the maximum of the right-hand side of (12) among all pairs of orthonormal, horizontal vectors $X, Y$ is reached, by the Schwarz inequality, for $Y= \pm T_{U}^{\sharp} X /\left\|T_{U}^{\sharp} X\right\|$. Note that $g\left(X, T_{U}^{\sharp} X\right)=g\left(\frac{1}{2} \mathcal{V}[X, X], U\right)=0$ and for all $X, Y$, such that $Y$ is orthogonal to both $X$ and $T_{U}^{\sharp} X$, both sides of (12) attain their minimum on the set of pairs of linearly independent vectors from $\mathcal{H}_{p}$. On the other hand, if $T_{U}^{\sharp} X=0$, then $S(X)=s(X)=e^{2 f}+2 e^{2 f} \phi-e^{2 f}\|\nabla f\|^{2}$.

Hence, by the above remark:

$$
S(X)=e^{2 f}+2 e^{2 f} \phi-e^{2 f}\|\nabla f\|^{2}+g\left(T_{U}^{\sharp} X, T_{U}^{\sharp} X\right),
$$

and for all $Y$ orthogonal to both $X$ and $T_{U}^{\sharp} X$, we have:

$$
\sec _{\mathrm{B}}\left(\pi_{*} X, \pi_{*} Y\right)=s(X)=e^{2 f}+2 e^{2 f} \phi-e^{2 f}\|\nabla f\|^{2} .
$$

It shows that $s=s(X)$ in fact does not depend on $X \in \mathcal{H}_{p}$. If $T_{U}^{\sharp} X=0$, we obtain that $S(X)=s(X)=s$. If $T_{U}^{\sharp} X \neq 0$, using the assumption that $\left(B, g_{\mathrm{B}}\right)$ is Einstein and taking an orthonormal basis $X_{n}$ of $\mathcal{H}_{p}$ where $X_{1}=X$ and $X_{2}=T_{U}^{\sharp} X /\left\|T_{U}^{\sharp} X\right\|$, we obtain:

$$
\lambda_{\mathrm{B}}=\operatorname{Ric}(X, X)=S(X)+(2 n-2) \cdot s,
$$

and it follows that $S=S(X)$ also does not depend on $X \in \mathcal{H}_{p}$. Comparing (14) and (15), we obtain for all unit horizontal vectors $X$ :

$$
S-s=3 e^{2 f}\left\|T_{U}^{\sharp} X\right\|^{2} .
$$

Note that from definition (13) and independence of $S$ and $s$ on $X$, it follows that:

$$
S=\max _{X, Y \in \mathcal{H}_{p}} \sec _{\mathrm{B}}\left(\pi_{*} X, \pi_{*} Y\right), \quad s=\min _{X, Y \in \mathcal{H}_{p}} \sec _{\mathrm{B}}\left(\pi_{*} X, \pi_{*} Y\right) .
$$

As $\pi_{*}$ is an isomorphism between $\mathcal{H}_{p}$ and $T_{\pi(p)} B$, it follows that $S-s$ is a function on $B$, constant on the fiber $\pi^{-1}(\{p\})$.

Assume that $T_{U}^{\sharp} \neq 0$ at $p \in M$. In what follows, we consider an orthonormal basis of $\mathcal{H}_{p}$, such that for all odd $1 \leq i<2 n=\operatorname{dim} B$, we have $X_{i+1}=T_{U}^{\sharp} X_{i} /\left\|T_{U}^{\sharp} X_{i}\right\|$.

For a unit vertical field $U$, we have:

$$
\begin{aligned}
\left\|T_{U}^{\sharp}\right\|^{2} & =\sum_{i, j=1}^{2 n} g\left(T_{U}^{\sharp} X_{i}, X_{j}\right)^{2} \\
& =\sum_{i=1}^{2 n} g\left(T_{U}^{\sharp} X_{i}, X_{i+1}\right)^{2} \\
& =\sum_{i=1}^{2 n} g\left(T_{U}^{\sharp} X_{i}, T_{U}^{\sharp} X_{i} /\left\|T_{U}^{\sharp} X_{i}\right\|\right)^{2} \\
& =\sum_{i=1}^{2 n} g\left(T_{U}^{\sharp} X_{i}, T_{U}^{\sharp} X_{i}\right)^{2} /\left\|T_{U}^{\sharp} X_{i}\right\|^{2} \\
& =\sum_{i=1}^{2 n} g\left(T_{U}^{\sharp} X_{i}, T_{U}^{\sharp} X_{i}\right)=6 n e^{-2 f}(S-s),
\end{aligned}
$$


where in the last line we used (16).

Let $U$ be a unit vertical vector field. We have for all horizontal vectors $X, Y$ :

$$
\left(\mathcal{L}_{U} g\right)(X, Y)=2 g(X, Y) U(f) .
$$

Since fibers are geodesic and $U$ is a unit field, we also have for all horizontal $X$ :

$$
\left(\mathcal{L}_{U} g\right)(X, U)=g\left(\nabla_{U} U, X\right)+g\left(\nabla_{X} U, U\right)=\frac{1}{2} X(g(U, U))=0,
$$

and

$$
\left(\mathcal{L}_{U} g\right)(U, U)=2 g\left(\nabla_{U} U, U\right)=U(g(U, U))=0 .
$$

Let $\phi_{t}$ denote the flow of $U$. We have [11]:

$$
\phi_{s}^{*}\left(\mathcal{L}_{\mathrm{U}} g\right)=\left.\frac{\mathrm{d}}{\mathrm{d} t}\left(\phi_{t}^{*} g\right)\right|_{t=s} .
$$

From (20) and $[U, U]=0$, it follows that $\phi_{t}$ preserves both horizontal and vertical distribution. Hence, for $X, Y \in \mathcal{H}_{p}$, we have:

$$
\begin{aligned}
\left.\frac{\mathrm{d}}{\mathrm{d} t}\left(\phi_{t}^{*} g(X, Y)\right)\right|_{t=s} & =\phi_{s}^{*}\left(\mathcal{L}_{U} g(X, Y)\right) \\
& =\phi_{s}^{*}(2 g(X, Y) U(f)) \\
& =2\left(\phi_{s}^{*} g\right)(X, Y) \cdot\left(U(f) \circ \phi_{s}\right) \\
& =\left.2\left(\phi_{s}^{*} g\right)(X, Y) \cdot \frac{\mathrm{d}}{\mathrm{d} t}\left(f \circ \phi_{t}\right)\right|_{t=s} .
\end{aligned}
$$

Solving the above ODE, we obtain:

$$
\left(\phi_{s}^{*} g\right)(X, Y)=g(X, Y) e^{2\left(f \circ \phi_{s}-f\right)},
$$

which we can write as:

$$
g\left(\phi_{s *} X, \phi_{s *} Y\right)=g(X, Y) e^{2\left(f \circ \phi_{s}-f\right)} .
$$

On the other hand, (21) implies that:

$$
g\left(\phi_{S *} U, \phi_{S *} U\right)=g(U, U) .
$$

Let $X_{i}$ be an orthonormal basis of $\mathcal{H}_{p}$. Then, from (22), it follows that vectors:

$$
Y_{i}=e^{-\left(f\left(\phi_{s}(p)\right)-f(p)\right)} \phi_{s *} X_{i}
$$

form an orthonormal basis at $\mathcal{H}_{\phi_{s}(p)}$ and by (23) $\phi_{s *} U$ is unit. The norm of $T_{U}^{\sharp}$ at $\phi_{s}(p)$ can be computed using $Y_{i}$, and thus equals to: 


$$
\begin{aligned}
\left\|T_{U}^{\sharp}\right\|_{\phi_{s}(p)}^{2} & =\sum_{i, j=1}^{2 n} g\left(T_{U}^{\sharp} Y_{i}, Y_{j}\right)^{2} \\
& =\sum_{i, j=1}^{2 n} g\left(T_{\phi_{s *}}^{\sharp} e^{-\left(f\left(\phi_{s}(p)\right)-f(p)\right)} \phi_{s *} X_{i}, e^{-\left(f\left(\phi_{s}(p)\right)-f(p)\right)} \phi_{s *} X_{j}\right)^{2} \\
& =\sum_{i, j=1}^{2 n} e^{-4\left(f\left(\phi_{s}(p)\right)-f(p)\right)} g\left(T_{\phi_{s *} U}^{\sharp} \phi_{s *} X_{i}, \phi_{s *} X_{j}\right)^{2} \\
& =\sum_{i, j=1}^{2 n} e^{-4\left(f\left(\phi_{s}(p)\right)-f(p)\right)} g\left(\frac{1}{2}\left[\phi_{s *} X_{i}, \phi_{s *} X_{j}\right], \phi_{s *} U\right)^{2} \\
& =\sum_{i, j=1}^{2 n} e^{-4\left(f\left(\phi_{s}(p)\right)-f(p)\right)} g\left(\frac{1}{2} \phi_{s *}\left[X_{i}, X_{j}\right], \phi_{s *} U\right)^{2} \\
& =\sum_{i, j=1}^{2 n} e^{-4\left(f\left(\phi_{s}(p)\right)-f(p)\right)} g\left(\frac{1}{2}\left[X_{i}, X_{j}\right], U\right)^{2} \\
& =\sum_{i, j=1}^{2 n} e^{-4\left(f\left(\phi_{s}(p)\right)-f(p)\right)} g\left(T_{U}^{\sharp} X_{i}, X_{j}\right)^{2} \\
& =\sum_{i, j=1}^{2 n} e^{-4\left(f\left(\phi_{s}(p)\right)-f(p)\right)}\left\|T_{U}^{\sharp}\right\|_{p}^{2} .
\end{aligned}
$$

Hence, if $T_{U}^{\sharp} X \neq 0$ for some $X \in \mathcal{H}_{p}$, then along the fiber through $p$, we have $\left\|T_{U}^{\sharp}\right\|^{2}=c e^{-4 f}$ for some $c>0$. From (18), we obtain:

$$
c e^{-4 f}=6 n e^{-2 f}(S-s)
$$

along the fiber through $p$, and it follows that $f$ is constant along all fibers through points where $T_{U}^{\sharp} \neq 0$.

If $T_{U}^{\sharp}=0$ at $p \in M$, then from (24), it follows that $T_{U}^{\sharp}=0$ along the fiber through $p$. Let $N \subset B$ be the set of points $q \in B$, such that $T_{U}^{\sharp}=0$ on the fiber $\pi^{-1}(\{q\})$. We will show that $f$ is constant along fibers over $N$.

Suppose that $q$ is an interior point of $N$, i.e., there exists an open set $V \subset N$ with $q \in V$. Then, $W=\pi^{-1}(V)$ is an open subset of $S^{2 n+1}$, and at every point $p \in W$, we have $T_{U}^{\sharp}=0$. But then, the horizontal distribution $\mathcal{H}$ of $\pi$ is integrable on the open set $W$, and so on $W$, there exists a foliation $\mathcal{F}$ tangent to $\mathcal{H}$. As $\pi$ is a conformal fibration by geodesics, $\mathcal{F}$ is a codimension one Riemannian foliation of $W$ with all leaves totally umbilical, orthogonal to a disjoint family of great circles. As $W \subset S^{2 n+1}$, leaves of $\mathcal{F}$ must be intersections of $S^{2 n+1}$ with parallel $(2 n+1)$-dimensional planes, and, hence, cannot be orthogonal to any disjoint family of great circles. Thus, set $N$ has empty interior.

If $q \in N$, then it is not an interior point of $N$, and hence, there exists a sequence $q_{n} \rightarrow q$, where $q_{n} \notin N$. As we proved above, $f$ is constant along all the fibers $\pi^{-1}\left(\left\{q_{n}\right\}\right)$, and by continuity of $f$, it must be constant along the fiber $\pi^{-1}(\{q\})$. Therefore, $f$ is constant along the fibers over $N$, and, hence, along all fibers of $\pi$.

Corollary 1 Let $\pi:\left(S^{2 n+1}, g\right) \rightarrow\left(B, g_{\mathrm{B}}\right), n>1$, be a conformal submersion with fibers being geodesics and let $\left(B, g_{\mathrm{B}}\right)$ be an Einstein manifold. Then, there exist isometries 
$\Lambda:\left(\mathbb{C} P^{n}, g_{\mathrm{FS}}\right) \rightarrow\left(B, g_{\mathrm{B}}\right), \Upsilon:\left(S^{2 n+1}, g\right) \rightarrow\left(S^{2 n+1}, g\right)$ and a conformal diffeomorphism $\Phi$ of $\mathbb{C} P^{n}$, such that $\pi=\Lambda \circ \Phi \circ \pi_{\mathrm{H}} \circ \Upsilon$, where $\pi_{\mathrm{H}}$ is the Hopf fibration and $g_{\mathrm{FS}}$ is the Fubini-Study metric.

Proof From Proposition 1, it follows that $f=h \circ \pi$ for some function $h \in C^{\infty}(B)$. Considering metric $g_{h}=e^{-2 h} g_{\mathrm{B}}$ on $B$, we obtain a Riemannian submersion $\pi:\left(S^{2 n+1}, g\right) \rightarrow\left(B, g_{h}\right)$, and by the classification from [4], it follows that there exist isometries $\Psi:\left(\mathbb{C} P^{n}, g_{\mathrm{FS}}\right) \rightarrow\left(B, g_{\mathrm{h}}\right)$ and $\Upsilon:\left(S^{2 n+1}, g\right) \rightarrow\left(S^{2 n+1}, g\right)$, such that $\pi=\Psi \circ \pi_{\mathrm{H}} \circ \Upsilon$.

Moreover, as $\Psi^{-1}$ is an isometry, $g_{\mathrm{h}}=\left(\Psi^{-1}\right)^{*} g_{\mathrm{FS}}$ is an Einstein metric on $B$. As metrics $g_{\mathrm{h}}$ and $g_{\mathrm{B}}$ are conformal and both have constant scalar curvatures, it follows from [17] that there exists an isometry $\Xi:\left(B, g_{\mathrm{h}}\right) \rightarrow\left(B, g_{\mathrm{B}}\right)$ and since $g_{\mathrm{h}}=e^{-2 h} g_{\mathrm{B}}, \Xi$ is a conformal diffeomorphism of $\left(B, g_{\mathrm{h}}\right)$. Let $\Phi=\Psi^{-1} \circ \Xi^{-1} \circ \Psi$, then $\Phi$ is a conformal diffeomorphism of $\left(\mathbb{C} P^{n}, g_{\mathrm{FS}}\right)$, and we have:

$$
\pi=\Psi \circ \pi_{\mathrm{H}}=\Xi \circ \Xi^{-1} \circ \Psi \circ \pi_{\mathrm{H}}=\Xi \circ \Psi \circ \Phi \circ \pi_{\mathrm{H}} .
$$

It remains to show that $\Lambda=\Xi \circ \Psi$ is an isometry from $\left(\mathbb{C} P^{n}, g_{\mathrm{FS}}\right)$ to $\left(B, g_{\mathrm{B}}\right)$. Indeed:

$$
\Lambda^{*} g_{\mathrm{B}}=(\Xi \circ \Psi)^{*} g_{\mathrm{B}}=\Psi^{*} \Xi^{*} g_{\mathrm{B}}=\Psi^{*} g_{h}=g_{\mathrm{FS}} \text {. }
$$

Clearly, the same argument applies for submersions $\pi:\left(S^{2 n+1}, g\right) \rightarrow\left(B, e^{2 \psi} g_{\mathrm{B}}\right), n>1$, where $\psi \in C^{\infty}(B)$ and $g_{\mathrm{B}}$ is an Einstein metric. In fact, we have the following.

Corollary 2 Let $\pi:\left(S^{2 n+1}, g\right) \rightarrow\left(B, g_{\mathrm{B}}\right), n>1$, be a conformal submersion with fibers being geodesics, and let $\left(B, g_{\mathrm{B}}\right)$ be conformal to an Einstein manifold. Then, $\pi=\Phi \circ \pi_{H} \circ \Upsilon$, where $\pi_{\mathrm{H}}$ is the Hopf fibration, $\Upsilon$ is an isometry of $\left(S^{2 n+1}, g\right), \Phi:\left(\mathbb{C} P^{n}, g_{\mathrm{FS}}\right) \rightarrow\left(B, g_{\mathrm{B}}\right)$ is a conformal diffeomorphism, and $g_{\mathrm{FS}}$ is the Fubini-Study metric.

Proof The proof follows from Corollary 1 for an Einstein manifold $\left(B, g_{\mathrm{E}}\right)$ conformal to $\left(B, g_{\mathrm{B}}\right)$, by composing the map $\Lambda \circ \Phi \circ \pi_{H} \circ \Upsilon$ obtained there with the conformal map from $\left(B, g_{\mathrm{E}}\right)$ to $\left(B, g_{\mathrm{B}}\right)$.

\section{Curvature of the image of a conformal submersion with minimal fibers}

Unlike Riemannian submersions [16], conformal submersion do not yield any obvious pointwise estimates for curvatures of their images. The relations between curvatures of the domain and the image of a conformal submersion contain the dilation of the submersion and its (first and second) derivatives, which can be made arbitrary at a single point by composing given submersion with a conformal diffeomorphism of its image. This part of the paper continues author's previous work [24] to obtain some global curvature conditions for existence of conformal submersions with fibers of particular geometry.

Let $\pi:(M, g) \rightarrow\left(B, g_{\mathrm{B}}\right)$ be a conformal submersion with minimal fibers. Recall the notation $b=\operatorname{dim} B$. Using the formula from [21], we can express the mixed scalar curvature $S_{\text {mix }}$ in the following way: 


$$
S_{\text {mix }}=-\|h\|^{2}-b \Delta^{\mathcal{V}} f+\sum_{i, j=1}^{b}\left\|A_{X_{j}} X_{i}\right\|^{2}-2 b\|\mathcal{V} \nabla f\|^{2},
$$

where $h$ is the second fundamental form of the fibers (see Definition 3) and:

$$
\|h\|^{2}=\sum_{i, j=1}^{k} g\left(h\left(U_{i}, U_{j}\right), h\left(U_{i}, U_{j}\right)\right)
$$

for any orthonormal basis $\left\{U_{1}, \ldots, U_{k}\right\}$ of the vertical distribution.

Let $\left\{X_{1}, \ldots X_{\mathrm{b}}\right\}$ be an orthonormal (with respect to $g$ ) basis of the horizontal distribution of $\pi$. Then, from (5), which holds for any conformal submersion, we obtain:

$$
\begin{aligned}
K_{\mathcal{H}}= & -2(b-1) \sum_{i=1}^{\mathrm{b}} \operatorname{Hess} f\left(X_{i}, X_{i}\right)+b(b-1)\|\nabla f\|^{2} \\
& -2(b-1)\|\mathcal{H} \nabla f\|^{2}+e^{-2 f}\left(K_{\mathrm{B}} \circ \pi\right) \\
& -3 \sum_{i, j=1}^{b}\left\|A_{X_{i}} X_{j}\right\|^{2}+3 b\|\mathcal{V} \nabla f\|^{2} .
\end{aligned}
$$

For minimal fibers and an orthonormal basis of the vertical distribution $\left\{U_{1}, \ldots, U_{k}\right\}$, we have:

$$
\begin{aligned}
\sum_{i=1}^{b} \operatorname{Hess} f\left(X_{i}, X_{i}\right)= & \Delta f-\sum_{j=1}^{k} g\left(\nabla_{U_{j}} \nabla f, U_{j}\right) \\
& =\Delta f-\Delta^{\mathcal{V}} f-\sum_{j=1}^{k} g\left(\nabla_{U_{j}} \mathcal{H} \nabla f, U_{j}\right) \\
& =\Delta f-\Delta^{\mathcal{V}} f+\sum_{j=1}^{k} g(H, \mathcal{H} \nabla f) g\left(U_{j}, U_{j}\right) \\
& =\Delta f-\Delta^{\mathcal{V}} f .
\end{aligned}
$$

Using the above in (26), we obtain the following.

Proposition 2 Let $\pi:(M, g) \rightarrow\left(B, g_{\mathrm{B}}\right)$ be a conformal submersion with minimal fibers and let $\left\{X_{1}, \ldots, X_{\mathrm{b}}\right\}$ be an orthonormal basis of the horizontal distribution on $M$. Then, the following equality holds:

$$
\begin{aligned}
K_{\mathcal{H}}= & e^{-2 f}\left(K_{\mathrm{B}} \circ \pi\right)-3 \sum_{i, j=1}^{b}\left\|A_{X_{i}} X_{j}\right\|^{2} \\
& -2(b-1) \Delta f+2(b-1) \Delta^{\mathcal{V}} f \\
& +(b-1)(b-2)\|\mathcal{H} \nabla f\|^{2}+b(b+2)\|\mathcal{V} \nabla f\|^{2},
\end{aligned}
$$

where $\Delta$ denotes the Laplacian on $M$.

Using similar formulas, some existence conditions were formulated for conformal submersions with totally umbilical fibers [24], in particular the following. 
Proposition 3 Let $M$ be a closed manifold with non-positive sectional curvature. Then, there exist no conformal submersions with totally umbilical fibers from $M$ onto a non-flat manifold of non-negative scalar curvature.

Below, we prove an analogous result: with the opposite signs of considered curvatures and for conformal submersions with minimal fibers.

Lemma 2 Let $\pi:(M, g) \rightarrow\left(B, g_{\mathrm{B}}\right)$ be a conformal submersion with minimal fibers. For any integer $c>1$, the following differential operator acting on $C^{\infty}(M)$ is elliptic:

$$
\mathcal{L}_{c}=-2(c-1) \Delta-(c+2) \Delta^{\mathcal{V}} .
$$

If $b>2$, we have:

$$
\begin{aligned}
-\frac{1}{b-2} e^{(b-2) f} \mathcal{L}_{b} e^{-(b-2) f}= & K_{\mathcal{H}}+3 S_{\text {mix }}+3\|h\|^{2}-e^{-2 f}\left(K_{\mathrm{B}} \circ \pi\right) \\
& +4(b-1)\|\mathcal{V} \nabla f\|^{2} \\
& +(b-1)(b-2)\|\nabla f\|^{2}
\end{aligned}
$$

and if $b=2$, we have:

$$
\begin{aligned}
\mathcal{L}_{2} f= & K_{\mathcal{H}}+3 S_{\text {mix }}+3\|h\|^{2}-e^{-2 f}\left(K_{\mathrm{B}} \circ \pi\right) \\
& +4\|\mathcal{V} \nabla f\|^{2} .
\end{aligned}
$$

Proof Suppose that $\pi:(M, g) \rightarrow\left(B, g_{\mathrm{B}}\right)$ is a conformal submersion with minimal fibers and assume that $b>1$. Then, (25) and (27) yield:

$$
\begin{aligned}
K_{\mathcal{H}}+3 S_{\text {mix }}+3\|h\|^{2}-e^{-2 f}\left(K_{\mathrm{B}} \circ \pi\right)= & -2(b-1) \Delta f-(b+2) \Delta^{\mathcal{V}} f \\
& +(b-1)(b-2)\|\mathcal{H} \nabla f\|^{2} \\
& +b(b-4)\|\mathcal{V} \nabla f\|^{2} .
\end{aligned}
$$

Since the fibers are minimal, we have at any point $p \in M$ :

$$
\Delta^{\mathcal{V}} f=\sum_{i=1}^{k} \operatorname{Hess} f\left(U_{i}, U_{i}\right)
$$

where vectors $U_{i}, i \in\{1, \ldots, k\}$ form an orthonormal basis of the vertical subspace at $p$. Hence, for all $\phi \in C^{\infty}(M)$ :

$$
\mathcal{L}_{b} \phi=-2(b-1) \sum_{j=1}^{b} \operatorname{Hess} \phi\left(X_{j}, X_{j}\right)-3 b \sum_{i=1}^{k} \operatorname{Hess} \phi\left(U_{i}, U_{i}\right),
$$

where vectors $U_{i}, i \in\{1, \ldots, k\}$ form an orthonormal basis of the vertical subspace and vectors $X_{j}, j \in\{1, \ldots, b\}$ form an orthonormal basis of the horizontal subspace. From (31), it follows that operators $\mathcal{L}_{b}$ are elliptic for $b>1$. Using the fact that for all vectors $X \in T M$, we have:

$$
\operatorname{Hess} e^{-(b-2) f}(X, X)=-(b-2) e^{-(b-2) f} \operatorname{Hess} f(X, X)+(b-2)^{2} e^{-(b-2) f}(X(f))^{2},
$$

and rearranging terms of (30), we eventually obtain (28) for $b>2$ and (29) for $b=2$. 
Proposition 4 Let $(M, g)$ be a closed Riemannian manifold of non-negative sectional curvature and let $\left(B, g_{\mathrm{B}}\right)$ be a closed Riemannian manifold of non-positive scalar curvature. If there exists a conformal submersion $\pi$ with minimal fibers from $(M, g)$ onto $\left(B, g_{\mathrm{B}}\right)$, then:

1. $\left(B, g_{\mathrm{B}}\right)$ is a flat manifold.

2. $(M, g)$ is locally a metric product of a flat manifold and a manifold of non-negative curvature.

3. If $b>1$, submersion $\pi$ has constant dilation.

4. If $b=1$, submersion $\pi$ has dilation constant along fibers.

Proof By the assumptions on the curvatures of $(M, g)$ and $\left(B, g_{\mathrm{B}}\right)$ for $b>2$, we have from (28):

$$
-\frac{1}{b-2} e^{(b-2) f} \mathcal{L}_{b} e^{-(b-2) f} \geq 0
$$

and for $b=2,(29)$ yields:

$$
\mathcal{L}_{2} f \geq 0
$$

Operators $-\mathcal{L}_{b}$ for $b>1$ are elliptic and vanish on constant functions. Hence, they satisfy the strong maximum principle [5] and from inequality (32) or (33) and the assumption that $M$ is closed, we obtain that $f=$ const, and $\pi:(M, g) \rightarrow\left(B, e^{2 f} \cdot g_{\mathrm{B}}\right)$ is a Riemannian submersion. From the results on Riemannian submersions [4] - or (30) for $f=0$-it follows that $\left(B, e^{2 f} \cdot g_{\mathrm{B}}\right)$ is flat, and hence, so is $\left(B, g_{\mathrm{B}}\right)$, as $f=$ const; fibers of $\pi$ are totally geodesic, i.e., $\|h\|=0$, and all sectional curvatures on $(M, g)$ of planes with at least one horizontal direction vanish.

The fundamental equation [16] for the Riemannian submersion $\pi:(M, g) \rightarrow\left(B, e^{2 f} \cdot g_{\mathrm{B}}\right)$ for horizontal, orthonormal vectors $X$ and $Y$ yields

$$
\sec (X, Y)=\sec _{\mathrm{B}}\left(\pi_{*} X, \pi_{*} Y\right)-3\left\|A_{X} Y\right\|^{2},
$$

from which it follows that $A=0$ and the horizontal distribution is integrable and totally geodesic. Since the fibers of the submersion are totally geodesic, $(M, g)$ is locally a metric product [8] of a flat manifold and a manifold of non-negative curvature.

For $b=1$, note that for a unit vector $X$ in the only horizontal direction, we have:

$$
\left\|A_{X} X\right\|^{2}=\|\mathcal{V} \nabla f\|^{2}
$$

Equation (25) yields:

$$
\Delta^{\mathcal{V}} f=-S_{\text {mix }}-\|\mathcal{V} \nabla f\|^{2} \leq 0,
$$

and it follows that the dilation $f$ is constant along the fibers of $\pi$, which implies in (34) that $A=0$. Again, we can consider Riemannian submersion $\pi:(M, g) \rightarrow\left(B, e^{2 f} \cdot g_{\mathrm{B}}\right)$ with 1-dimensional (and hence integrable) geodesic horizontal distribution.

Corollary 3 There are no conformal submersions with minimal fibers from a closed manifold of non-negative curvature onto a non-flat manifold with non-positive scalar curvature.

Together with Proposition 3, Corollary 3 yields the following. 
Corollary 4 Let $(M, g)$ be a closed Riemannian manifold and let $\pi:(M, g) \rightarrow\left(B, g_{\mathrm{B}}\right)$ be a conformal submersion with totally geodesic fibers. If the sectional curvature of $(M, g)$ and the scalar curvature of $\left(B, g_{\mathrm{B}}\right)$ are of constant signs, then they are of the same sign.

If $\pi:(M, g) \rightarrow\left(B, g_{\mathrm{B}}\right)$ is a conformal submersion, then $\pi:(M, g) \rightarrow\left(B, e^{2 \psi} g_{\mathrm{B}}\right)$ is a conformal submersion for every $\psi \in C^{\infty}(B)$. For $K_{\mathrm{B}}<0$, inequalities (32), (33) become sharp and thus cannot be satisfied by any $f \in C^{\infty}(M)$ on a closed manifold $M$. Hence, we obtain the following result.

Corollary 5 Let $\left(B, g_{\mathrm{B}}\right)$ be a closed Riemannian manifold. If, in the conformal class of $g_{\mathrm{B}}$, there exists a metric with negative scalar curvature, then there are no conformal submersions with minimal fibers from a closed manifold $(M, g)$ onto $\left(B, g_{\mathrm{B}}\right)$, such that $K_{\mathcal{H}}+3 S_{\text {mix }} \geq 0$. In particular, there are no conformal submersions with minimal fibers onto $\left(B, g_{\mathrm{B}}\right)$ from closed manifolds with non-negative sectional curvature.

Note that from the existence of solutions to Yamabe problem, it follows that every class of conformal metrics has a metric of constant scalar curvature. Thus, the above corollary limits conformal classes of Riemannian manifolds that may be images of certain conformal submersions.

Open Access This article is licensed under a Creative Commons Attribution 4.0 International License, which permits use, sharing, adaptation, distribution and reproduction in any medium or format, as long as you give appropriate credit to the original author(s) and the source, provide a link to the Creative Commons licence, and indicate if changes were made. The images or other third party material in this article are included in the article's Creative Commons licence, unless indicated otherwise in a credit line to the material. If material is not included in the article's Creative Commons licence and your intended use is not permitted by statutory regulation or exceeds the permitted use, you will need to obtain permission directly from the copyright holder. To view a copy of this licence, visit http://creativecommons.org/licenses/by/4.0/.

\section{References}

1. Aubin, T.: Equations differentielles non lineaires et probleme de Yamabe concernant la courbure scalaire. J. Math. Pures Appl. 55, 269-296 (1976)

2. Besse, A.L.: Einstein Manifolds. Springer-Verlag, Berlin (1987)

3. Ehresmann, C.: Les connexions infinitesimales dans un espace fibre differentiable, Colloque de topologie (espaces fibres), Bruxelles, 1950, Georges Thone, pp. 29-55. Liege; Masson et Cie, Paris (1951)

4. Escobales Jr., R.H.: Riemannian submersions with totally geodesic fibers. J. Differ. Geom. 10(2), 253$276(1975)$

5. Evans, L.C.: Partial Differential Equations. AMS, Providence (1998)

6. Gluck, H., Warner, F.W.: Great circle fibrations of the three-sphere. Duke Math. J. 50, 107-132 (1983)

7. Gluck, H., Warner, F., Yang, C.T.: Division algebras, fibrations of spheres by great spheres and the topological determination of space by the gross behavior of its geodesics. Duke Math. J. 50, 10411076 (1983)

8. Gromoll, D., Walschap, G.: Metric Foliations and Curvature. Birkhauser, Basel (2009)

9. Gudmundsson, S.: On the geometry of harmonic morphisms. Math. Proc. Camb. Philos. Soc. 108, 461-466 (1990)

10. Heller, S.G.: Conformal fibrations of $S^{3}$ by circles. In: Loubeau, E., Montaldo, S. (eds.) Harmonic Maps and Differential Geometry, pp. 195-202, Contemp. Math. 542, AMS, Providence (2011)

11. Kobayashi, S., Nomizu, N.: Foundations of Differential Geometry I. Interscience, New York (1963)

12. McKay, B.: The Blaschke conjecture and great circle fibrations of spheres. Am. J. Math. 126(5), 11551191 (2004) 
13. McKay, B.: The Blaschke conjecture and great circle fibrations of spheres, arXiv:math/0112027 [math. DG]

14. Naveira, A.M.: A classification of Riemannian almost-product manifolds. Rend. Mat. 7(3), 577-592 (1983)

15. Cahn, P., Gluck, H., Nuchi, H.: Germs of fibrations of spheres by great circles always extend to the whole sphere. Algebra Geom. Topol. 18, 1323-1360 (2018)

16. O'Neill, B.: The fundamental equations of a submersion. Mich. Math. J. 13, 459-469 (1966)

17. Obata, M.: Certain conditions for a Riemannian manifold to be isometric with a sphere. J. Math. Soc. Jpn. 14, 333-340 (1962)

18. Ranjan, A.: Riemannian submersions of spheres with totally geodesic fibers. Osaka J. Math. 22, 243260 (1985)

19. Schoen, R.: Conformal deformation of a Riemannian metric to constant scalar curvature. J. Differ. Geom. 20, 479-495 (1984)

20. Trudinger, N.: Remarks concerning the conformal deformation of Riemannian structures on compact manifolds. Ann. Scuola Norm. Sup. Pisa 22, 265-274 (1968)

21. Walczak, P.: An integral formula for a Riemannian manifold with two orthogonal complementary distributions. Colloq. Math. 58, 243-252 (1990)

22. Wilking, B.: Index parity of closed geodesics and rigidity of Hopf fibrations. Invent. Math. 144, 281295 (2001)

23. Yamabe, H.: On a deformation of Riemannian structures on compact manifolds. Osaka Math. J. 12, 21-37 (1960)

24. Zawadzki, T.: Existence conditions for conformal submersions with totally umbilical fibers. Differ. Geom. Appl. 35, 69-85 (2014)

Publisher's Note Springer Nature remains neutral with regard to jurisdictional claims in published maps and institutional affiliations. 